Editorial

\title{
Two-Dimensional Electronics and Optoelectronics: Present and Future
}

\author{
Zhixian Zhou ${ }^{1, *}$ and Yoke Khin Yap ${ }^{2, *}$ \\ 1 Department of Physics \& Astronomy, Wayne State University, Detroit, MI 48201, USA \\ 2 Department of Physics, Michigan Technological University, Houghton, MI 49931, USA \\ * Correspondence: zxzhou@wayne.edu (Z.Z.); ykyap@mtu.edu (Y.K.Y.); \\ Tel.: +1-313-577-2751 (Z.Z.); +1-906-487-2900 (Y.K.Y.)
}

Received: 18 July 2017; Accepted: 19 July 2017; Published: 22 July 2017

\section{Introduction}

Since the successful isolation of graphene a little over a decade ago, a wide variety of two-dimensional (2D) layered materials have been studied. They cover a broad spectrum of electronic properties, including metals, semimetals, semiconductors, and insulators. Many of these 2D materials have demonstrated promising potential for electronic and optoelectronic applications.

Graphene has attracted a tremendous amount of attention from the scientific community largely due to its combination of extremely high carrier mobility and thermal conductivity with mechanical strength and flexibility as well as high thermal and chemical stability [1-4]. However, the lack of a fundamental bandgap in graphene has significantly limited its applications in electronics and optoelectronics. The research community is now turning its attention to 2D materials beyond graphene, particularly 2D semiconductors with an appropriate bandgap such as transition metal dichalcogenides (TMDCs) and black phosphorus [5-8]. The interlayer van der Waal bonding in 2D materials also offers the opportunities to create a large number of heterostructures by artificially stacking different 2D materials together without the constraints of atomic commensurability.

\section{The Present Issue}

This special issue consists of seven papers covering important topics in the field of 2D materials, including two reviews focusing on electronic and optoelectronic devices based on 2D materials. The contents of these papers are introduced here.

In Reference [9], micro-reflectance spectroscopy is used to investigate the differential reflectance spectra of hundreds of $\mathrm{MoS}_{2}$ flakes grown on a highly-polished sapphire substrate by chemical vapor deposition. This fast and non-destructive characterization technique is able to measure a large number of spectra in different sample locations and in a small amount of time. The growth of smooth and continuous layers of $\gamma$-InSe and $\mathrm{Sb}_{2} \mathrm{Se}_{3}$ layered metal chalcogenide on $\mathrm{SiO}_{2}$-coated $\mathrm{Si}$ and glass substrates by atomic layer deposition (ALD) is described in Reference [10]. This work shows that ALD offers a viable path for producing large area films of metal chalcogenides for future industrial-scale applications. A first-principles study of the structural, energetic, and electronic properties of single-layer graphene doped with boron and nitrogen atoms with varying doping concentrations and configurations is described in Reference [11]. The calculations in the paper indicate that the bandgap can be adjusted as required based on the doping concentration and the doping configuration. The modeling and design of a new flexible graphene-on-silicon Schottky junction solar cell with a power conversion efficiency $>10 \%$ is described in Reference [12]. Reference [13] proposes asymmetric double-well potential on graphene as an electronic waveguide to confine the graphene electrons. The guided modes in this graphene waveguide are investigated using a modified transfer matrix method. 
An overview of recent advances in electronic and optoelectronic devices based on 2D TMDCs is presented in Reference [14]. This review focuses on evaluating field-effect transistors (FETs), photovoltaic cells, light-emitting diodes (LEDs), photodetectors, lasers, and integrated circuits (ICs) using TMDCs. The review "Photonic Structure-Integrated Two-Dimensional Material Optoelectronics" offers an overview and evaluation of state-of-the-art of hybrid systems, where 2D material optoelectronics are integrated with photonic structures, especially plasmonic nanostructures, photonic waveguides, and crystals [15].

\section{Future}

While the potential of 2D materials in future flexible electronics and optoelectronics has been widely recognized by the scientific community, several major challenges still remain. The lack of effective methods to $p$ - and $n$-dope 2D semiconductors has seriously restricted their device applications. The presence of a significant contact barrier between most 2D semiconductors and common electrode materials has so far limited the performance of 2D electronics and prevented the ultimate downscaling of the device dimensions. Techniques to grow high quality 2D materials with wafer-scale uniformity need to be developed to scale up the production of 2D electronic and optoelectronic devices. The low carrier mobility in 2D semiconductors appears to limit their advantages over the current technology. Future innovations that overcome these bottlenecks will likely lead to breakthroughs in 2D electronics and optoelectronics. New 2D materials with promising electronic and optoelectronic properties are also likely to emerge in the future.

Acknowledgments: First of all we would like to thank all researchers who submitted articles to this special issue for their excellent contributions. We are also grateful to all reviewers who helped in the evaluation of the manuscripts and made very valuable suggestions to improve the quality of contributions. We would like to acknowledge the editorial board of Electronics, who invited us to guest edit this special issue. We are also grateful to the Electronics Editorial Office staff who worked thoroughly to maintain the rigorous peer-review schedule and timely publication. Y.K.Y. is supported by National Science Foundation (Award number DMR-1261910) during the course of editing this special issue.

Author Contributions: Z.Z. and Y.K.Y. worked together in the whole editorial process of the special issue, 'Two-Dimensional Electronics and Optoelectronics', published by journal Electronics. Z.Z. drafted this editorial summary. Z.Z. and Y.K.Y. reviewed, edited and finalized the manuscript.

Conflicts of Interest: The authors declare no conflicts of interest.

\section{References}

1. Balandin, A.A.; Ghosh, S.; Bao, W.; Calizo, I.; Teweldebrhan, D.; Miao, F.; Lau, C.N. Superior Thermal Conductivity of Single-Layer Graphene. Nano Lett. 2008, 8, 902-907. [CrossRef] [PubMed]

2. Ghosh, S.; Calizo, I.; Teweldebrhan, D.; Pokatilov, E.P.; Nika, D.L.; Balandin, A.A.; Bao, W.; Miao, F.; Lau, C.N. Extremely high thermal conductivity of graphene: Prospects for thermal management applications in nanoelectronic circuits. Appl. Phys. Lett. 2008, 92, 151911. [CrossRef]

3. Seol, J.H.; Jo, I.; Moore, A.L.; Lindsay, L.; Aitken, Z.H.; Pettes, M.T.; Li, X.; Yao, Z.; Huang, R.; Broido, D.; et al. Two-Dimensional Phonon Transport in Supported Graphene. Science 2010, 328, 213-216. [CrossRef] [PubMed]

4. Bolotin, K.I.; Sikes, K.J.; Jiang, Z.; Klima, M.; Fudenberg, G.; Hone, J.; Kim, P.; Stormer, H.L. Ultrahigh electron mobility in suspended grapheme. Solid State Commun. 2008, 146, 351-355. [CrossRef]

5. Radisavljevic, B.; Radenovic, A.; Brivio, J.; Giacometti, V.; Kis, A. Single-layer $\mathrm{MoS}_{2}$ transistors. Nat. Nanotechnol. 2011, 6, 147-150. [CrossRef] [PubMed]

6. Novoselov, K.S.; Jiang, D.; Schedin, F.; Booth, T.J.; Khotkevich, V.V.; Morozov, S.V.; Geim, A.K. Two-dimensional atomic crystals. Proc. Natl. Acad. Sci. USA 2005, 102, 10451-10453. [CrossRef] [PubMed]

7. Li, L.; Yu, Y.; Ye, G.J.; Ge, Q.; Ou, X.; Wu, H.; Feng, D.; Chen, X.H.; Zhang, Y. Black phosphorus field-effect transistors. Nat. Nanotechnol. 2014, 9, 372-377. [CrossRef] [PubMed]

8. Liu, H.; Neal, A.T.; Zhu, Z.; Luo, Z.; Xu, X.; Tománek, D.; Ye, P.D. Phosphorene: An Unexplored 2D Semiconductor with a High Hole Mobility. ACS Nanotechnol. 2014, 8, 4033-4041. [CrossRef] [PubMed] 
9. Ghasemi, F.; Frisenda, R.; Dumcenco, D.; Kis, A.; Perez de Lara, D.; Castellanos-Gomez, A. High Throughput Characterization of Epitaxially Grown Single-Layer $\mathrm{MoS}_{2}$. Electronics 2017, 6, 28. [CrossRef]

10. Browning, R.; Kuperman, N.; Moon, B.; Solanki, R. Atomic Layer Growth of InSe and $\mathrm{Sb}_{2} \mathrm{Se}_{3} \mathrm{Layered}$ Semiconductors and Their Heterostructure. Electronics 2017, 6, 27. [CrossRef]

11. Varghese, S.; Swaminathan, S.; Singh, K.; Mittal, V. Energetic Stabilities, Structural and Electronic Properties of Monolayer Graphene Doped with Boron and Nitrogen Atoms. Electronics 2016, 5, 91. [CrossRef]

12. Xu, Y.; Ang, L. Guided Modes in a Double-Well Asymmetric Potential of a Graphene Waveguide. Electronics 2016, 5, 87. [CrossRef]

13. Dell'Olio, F.; Palmitessa, M.; Ciminelli, C. Modeling and Design of a New Flexible Graphene-on-Silicon Schottky Junction Solar Cell. Electronics 2016, 5, 73. [CrossRef]

14. Ye, M.; Zhang, D.; Yap, Y. Recent Advances in Electronic and Optoelectronic Devices Based on Two-Dimensional Transition Metal Dichalcogenides. Electronics 2017, 6, 43.

15. Wang, T.; Xu, Y.-Q. Photonic Structure-Integrated Two-Dimensional Material Optoelectronics. Electronics 2016, 5, 93. [CrossRef]

(C) 2017 by the authors. Licensee MDPI, Basel, Switzerland. This article is an open access article distributed under the terms and conditions of the Creative Commons Attribution (CC BY) license (http:/ / creativecommons.org/licenses/by/4.0/). 